\title{
Estudio FT-IR de la superficie de óxidos mixtos de Fe y Ga mediante la adsorción de moléculas prueba
}

\author{
J. M. GALLARDO AMORES, ${ }^{1}$ V. SÁNCHEZ ESCRIBANO ${ }^{2}$, G. BUSCA ${ }^{3}$ \\ ${ }^{1}$ Lab. Altas Presiones. Dpto. Q. Inorgánica I, Universidad Complutense, Ciudad Universitaria, E-28040 Madrid. \\ ${ }^{2}$ Dpto. Q. Inorgánica, U. Salamanca, Pa Merced, E-37008 Salamanca. \\ ${ }^{3}$ Istituto di Chimica, Università di Genova, P.le J.F. Kennedy, I-16129 Génova (Italia).
}

\begin{abstract}
Se han sintetizado varias muestras de óxidos mixtos de Fe y Ga con estequiometría $\mathrm{Fe}_{1-\mathrm{x}} \mathrm{Ga}_{\mathrm{x}} \mathrm{O}$ y $(\mathrm{x}=0,0.20,0.25,0.50,0.80$, 0.90) mediante un método convencional de coprecipitación a $\mathrm{pH}$ controlado, secado a $90{ }^{\circ} \mathrm{C}$ y calcinación a 400 y $800{ }^{\circ} \mathrm{C}$ durante 3 horas. Todas las muestras se caracterizaron mediante difracción de rayos $\mathrm{X}$, análisis químico, análisis térmicos (TG-ATD), espectroscopías FT-IR y VIS-UV. Se encontró que las muestras formaban una solución sólida $\alpha$-( $\mathrm{Fe}, \mathrm{Ga})_{2} \mathrm{O}_{3}$ en todo el rango de composiciones $\mathrm{Fe} / \mathrm{Ga}$ hasta $400{ }^{\circ} \mathrm{C}$, produciéndose muestras bifásicas a partir de $800{ }^{\circ} \mathrm{C}$ para $\mathrm{x}>0.50$, debido a la distinta estructura de la $\beta-\mathrm{Ga}_{2} \mathrm{O}_{3}$ respecto a la $\alpha-\mathrm{Fe}_{2} \mathrm{O}_{3}$. La acidez se estudió mediante la adsorción de pivalonitrilo sobre pastillas prensadas del propio material, observándose acidez tipo Lewis débil no siendo posible distinguir ambos cationes y Brönsted que aumentaba con el contenido de Ga. La adsorción de la molécula de metanol mostró que existe una potenciación de la reactividad cuando se añaden pequeñas cantidades de Ga.
\end{abstract}

Palabras clave: espectroscopía FT-IR, óxido de galio, disolución sólida, pivalonitrilo y metanol

\section{A FT-IR study of the Fe Ga mixed oxide surface by probe molecule adsorption}

Several Fe Ga mixed oxide samples denoted as $\mathrm{Fe}_{1-\mathrm{x}} \mathrm{Ga}_{\mathrm{x}} \mathrm{O}_{\mathrm{y}}(\mathrm{x}=0,0.20,0.25,0.50,0.80,0.90)$ have been synthesised by a conventional coprecipitation method at controlled $\mathrm{pH}$, dried at $90{ }^{\circ} \mathrm{C}$ for 24 hours and calcined at 400 y $800{ }^{\circ} \mathrm{C}$ for 3 hours. All samples were characterised by $\mathrm{X}$-ray diffraction, chemical analyses and thermogravimetry and differential thermal analysis (TG-DTA) and FT-IR and UV-VIS spectroscopies. It was found all materials formed a solid solution, $\alpha-(\mathrm{Fe}, \mathrm{Ga})_{2} \mathrm{O}_{3}$, in the entire $\mathrm{Fe} / \mathrm{Ga}$ compositional range until $400{ }^{\circ} \mathrm{C}$. Samples calcined at $800{ }^{\circ} \mathrm{C}$ gave rise to biphasic samples for $\mathrm{x}>0.5$ due to different structure of the $\beta-\mathrm{Ga}_{2} \mathrm{O}_{3}$ with respect to the $\alpha-\mathrm{Fe}_{2} \mathrm{O}_{3}$ one. Surface acidity of samples was studied by pivalonitrile molecule adsorption on the pressed pellet of each material, founding weak Lewis acid in all cases and not being possible distinguish both cation sites on the surface and Brönsted one that increased by increasing Ga content. Methanol molecule was also adsorbed, showing an increase of reactivity for those samples with a little bit of Ga content.

Keywords: FT-IR spectroscopy, gallium oxide, solid solution, pivalonitrile and methanol.

\section{INTRODUCCIÓN}

En los últimos años el óxido de galio está siendo objeto de investigación, debido a sus conocidas propiedades deshidrogenativas de alcanos ligeros $(1,2,3)$ y al denominado proceso Cyclar que transforma alcanos de cadena $\mathrm{C}_{3}-\mathrm{C}_{5}$ en ciclos aromáticos e $\mathrm{H}_{2}(4)$. Los trabajos más recientes lo proponen como un catión de intercambio en la estructura de materiales zeolíticos, generador de centros ácidos Brönsted que mejoran sustancialmente la selectividad en muchas reacciones $(5,6)$. También ha sido utilizado en catálisis ambiental, en reacciones de eliminación de $\mathrm{NO}_{x}$ en presencia de hidrocarburos, mezclado mecánicamente con $\mathrm{Al}_{2} \mathrm{O}_{3}(7)$.

Teniendo en cuenta estos precedentes y los anteriores trabajos del grupo de investigación sobre sistemas mixtos trivalentes $(8,9)$, parecía razonable estudiar el efecto producido sobre el óxido de hierro. Por otra parte, debido a que estos cationes dan estructuras tipo corindón y a su similitud de radios ióni- cos, es posible que puedan producirse soluciones sólidas y establecerse un efecto sinérgico en las propiedades originadas por ambos cationes.

\section{PROCEDIMIENTO EXPERIMENTAL}

\subsection{Preparación de las muestras}

Los óxidos mixtos de $\mathrm{Fe}$ y $\mathrm{Ga}$ con fórmula general $\mathrm{Fe}_{1}$ ${ }_{x} \mathrm{Ga}_{\mathrm{x}} \mathrm{O}_{\mathrm{y}}(\mathrm{x}=0,0.10,0.20,0.50,0.80,0.90)$ se sintetizaron mediante un método de coprecipitación a $\mathrm{pH}$ controlado, partiendo de soluciones acuosas conteniendo las sales precursoras, $\mathrm{Fe}\left(\mathrm{NO}_{3}\right)_{2} \cdot 9 \mathrm{H}_{2} \mathrm{O}$ y $\mathrm{Ga}\left(\mathrm{NO}_{3}\right)_{3} \cdot 9 \mathrm{H}_{2} \mathrm{O}$, con las cantidades requeridas para obtener las anteriores composiciones y añadiendo bicarbonato amónico hasta fijar el $\mathrm{pH}$ a 8,5. Después de un envejecimiento de $24 \mathrm{~h}$, filtrar, lavar y secar a $90{ }^{\circ} \mathrm{C} 24 \mathrm{~h}$, las muestras se calcinaron a $400{ }^{\circ} \mathrm{C} 3 \mathrm{~h}$. 


\subsection{Técnicas experimentales}

Los análisis químicos se realizaron utilizando un espectrómetro de emisión Plasma II Perkin-Elmer después de disolver las muestras en una mezcla de $\mathrm{HF}-\mathrm{HNO}_{3}$. En todos los casos se encontró un buen acuerdo entre las composiciones teóricas y las experimentales.

Los difractogramas de rayos $\mathrm{X}$ se registraron en un difractómetro Philips PW 1710 (radiación $\mathrm{Cu} \mathrm{K \alpha}$ filtro $\mathrm{Ni}$; 45kV, 35mA).

Los análisis térmicos (DTA-TG) se realizaron, en atmósfera de aire, con una termobalanza Setaram TGA 92-12, desde temperatura ambiente hasta $1273 \mathrm{~K}$ a la velocidad de calentamiento de $10 \mathrm{~K} / \mathrm{min}$.

Los espectros FT-IR se registraron con un espectrómetro Nicolet Magna 750 con transformada de Fourier en la región 4000-50 $\mathrm{cm}^{-1}$. Los espectros FT-IR después de la adsorción de pivalonitrilo sobre discos prensados de las muestras previamente tratadas se registraron después de evacuación y calentamiento a diferentes temperaturas.

Los espectros de reflectancia difusa visible-ultravioleta se realizaron en un espectrofotómetro JASCO V-570 en el intervalo 200-2500 nm utilizando pastillas prensadas de las muestras y un polímero como referencia.

\section{RESULTADOS Y DISCUSIÓN}

\subsection{Caracterización de las muestras}

\subsubsection{DIFRACCIÓN DE RX}

En la fig. 1 se comparan los difractogramas de RX de las muestras calcinadas a $400{ }^{\circ} \mathrm{C}$. Las muestras de óxido hierro y galio puros (Fig. $1 \mathrm{a}, \mathrm{g}$ ) presentan unos difractogramas de materiales cuyas reflexiones corresponden totalmente con las fases $\alpha-\mathrm{Fe}_{2} \mathrm{O}_{3}$, hematita (ICDD, $\mathrm{n}^{\mathrm{o}}$ : 33-664) y $\alpha-\mathrm{Ga}_{2} \mathrm{O}_{3}$ (ICDD, $n^{\circ}:$ 6-503), respectivamente. Cuando se añade Ga los materiales decrecen significativamente la intensidad de las reflexiones hasta la muestra de composición Fe/ Ga $=3$ (Fig. 1,c). En este caso los picos llegan a ser notablemente más anchos y el fondo crece fuertemente. A partir de la muestra $\mathrm{Fe}_{1.0} \mathrm{Ga}_{1.0} \mathrm{O}_{\text {y }}$ de nuevo

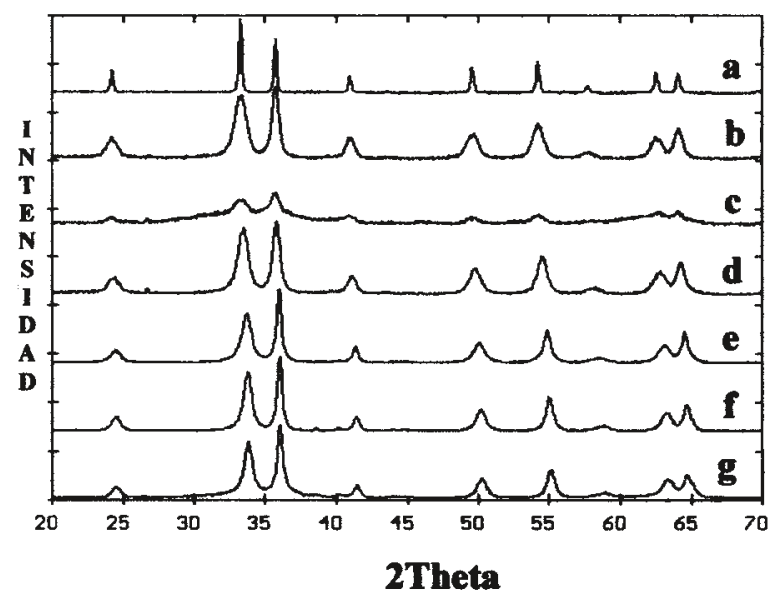

Fig. 1. Difractogramas de rayos $\mathrm{X}$ de las muestras calcinadas a 400 ${ }^{\circ} \mathrm{C}$. a) Fe2O3, b) Fe1.8Ga0.2Oy, c) Fe1.5Ga0.5Oy, d) Fe1.0Ga1.0Oy, e) $\mathrm{Fe} 0.5 \mathrm{Ga} 1.5 \mathrm{Oy}, \mathrm{f}) \mathrm{Fe} 0.2 \mathrm{Ga} 1.8 \mathrm{Oy}$ y g) $\mathrm{Ga}_{2} \mathrm{O}_{3}$.
TABLA I. PARÁMETROS DE CELDA Y VOLÚMENES DE LAS MUESTRAS CALCINADAS A $400{ }^{\circ} \mathrm{C}$.

\begin{tabular}{|c|c|c|c|c|}
\hline Muestra & Fase & \multicolumn{3}{|c|}{ Parámetros de celda } \\
\hline & \multirow{3}{*}{$\alpha-\mathrm{Fe}_{2} \mathrm{O}_{3}$} & $a=b(\AA)$ & c $(\AA ̊)$ & $\mathrm{V}\left(\AA^{3}\right)$ \\
\hline$\alpha-\mathrm{Fe}_{2} \mathrm{O}_{3}{ }^{\mathrm{a}}$ & & 5.035 & 13.74 & 301.9 \\
\hline $\mathrm{Fe}_{2} \mathrm{O}_{\mathrm{y}}$ & & $5.024(1)$ & $13.736(3)$ & 300.3 \\
\hline $\mathrm{Fe}_{1.8} \mathrm{Ga}_{0.2} \mathrm{O}_{\mathrm{y}}$ & \multirow{5}{*}{$\alpha-(\mathrm{Fe}, \mathrm{Ga})_{2} \mathrm{O}_{3}$} & $5.025(2)$ & $13.731(10)$ & 300.7 \\
\hline $\mathrm{Fe}_{1.5} \mathrm{Ga}_{0.5} \mathrm{O}_{\mathrm{y}}$ & & $4.993(1)$ & $13.709(20)$ & 295.9 \\
\hline $\mathrm{Fe}_{1.0} \mathrm{Ga}_{1.0} \mathrm{O}_{\mathrm{y}}$ & & $4.990(10)$ & $13.659(21)$ & 294.5 \\
\hline $\mathrm{Fe}_{0.5} \mathrm{Ga}_{1.5} \mathrm{O}_{\mathrm{y}}$ & & 4.998 (4) & $13.503(14)$ & 292.2 \\
\hline $\mathrm{Fe}_{0.2} \mathrm{Ga}_{1.8} \mathrm{O}_{\mathrm{y}}$ & & $4.987(2)$ & $13.475(6)$ & 290.3 \\
\hline $\begin{array}{l}\mathrm{Ga}_{2} \mathrm{O}_{\mathrm{y}} \\
\alpha-\mathrm{Ga}_{2} \mathrm{O}_{3} \mathrm{~b}\end{array}$ & $\alpha-\mathrm{Ga}_{2} \mathrm{O}_{3}$ & $\begin{array}{c}4.984(0) \\
4.979\end{array}$ & $\begin{array}{c}13.452(1) \\
13.42\end{array}$ & $\begin{array}{c}289.4 \\
288.3\end{array}$ \\
\hline
\end{tabular}

a. ICDD: $\mathrm{n}^{\circ}$ 33-664 (hematita sintética), b. ICDD: $\mathrm{n}^{\circ}$ 6-503 (a- $\mathrm{Ga}_{2} \mathrm{O}_{3}$ sintética)

se recupera la cristalinidad observándose un desplazamiento progresivo de las reflexiones hacia espaciados inferiores con el aumento del contenido de Ga. Los parámetros de celda y los volúmenes de los óxidos mixtos y puros se resumen en la Tabla I. Se observa una disminución progresiva de los parámetros de celda y de los volúmenes con el aumento del contenido de Ga que está de acuerdo con la evolución observada de los difractogramas. Estos resultados están indicando la formación de una solución sólida en todo el rango de composiciones Fe/ Ga con significativas variaciones de la cristalinidad que se puede denotar como $\alpha-(\mathrm{Fe}, \mathrm{Ga})_{2} \mathrm{O}_{3}$. En anteriores trabajos (10), este hecho se había observado para hidróxidos mixtos de Fe y Ga y en parte se atribuyó al carácter isoestructural de ambos óxidos (estructura tipo corindón) y a la similitud de sus radios iónicos en coordinación octaédrica (11). Un comportamiento parecido presenta el sistema de óxidos mixtos de Fe y $\mathrm{Cr}$ (12), aunque en este caso la cristalinidad de los materiales es notablemente menor por la presencia del $\mathrm{Cr}$ (13).

De acuerdo con los análisis térmicos y las calcinaciones realizadas a $800{ }^{\circ} \mathrm{C}$ que no se reflejan explícitamente, estas disoluciones sólidas son estables hasta $800{ }^{\circ} \mathrm{C}$ y una relación de composiciones $\mathrm{Fe} / \mathrm{Ga}=3$. A partir de estos límites el carácter termodinámicamente más estable de la fase $\beta-\mathrm{Ga}_{2} \mathrm{O}_{3}$ se pone de manifiesto, encontrándose materiales bifásicos en los difractogramas de rayos $\mathrm{X}$.

\subsubsection{ESPECTROSCOPÍA INFRARROJA POR TRANSFOR- MADA DE FOURIER.}

Los espectros FT-IR del material sin Ga muestran bandas de absorción bien definidas próximas a 233, 303, 392, 444 y 535 $\mathrm{cm}^{-1}$ y dos hombros a 667 y $691 \mathrm{~cm}^{-1}$ (Fig. 2,a) asignadas a $\alpha-\mathrm{Fe}_{2} \mathrm{O}_{3}$ en buen acuerdo con los espectros aportados en trabajos previos $(14,15)$. La adición de Ga produce el desplazamiento de todas las bandas hacia mayores números de ondas, cambia significativamente su forma siendo más amplias y las menos intensas no se distinguen en el espectro del material $\mathrm{Fe}_{1.5} \mathrm{Ga}_{0.5} \mathrm{O}_{\text {y }}$ (Fig. 2,c) de acuerdo con la pérdida de cristalinidad observada en los difractogramas. Un efecto similar se observó por Prieto y col. en el sistema de óxidos mixtos de 


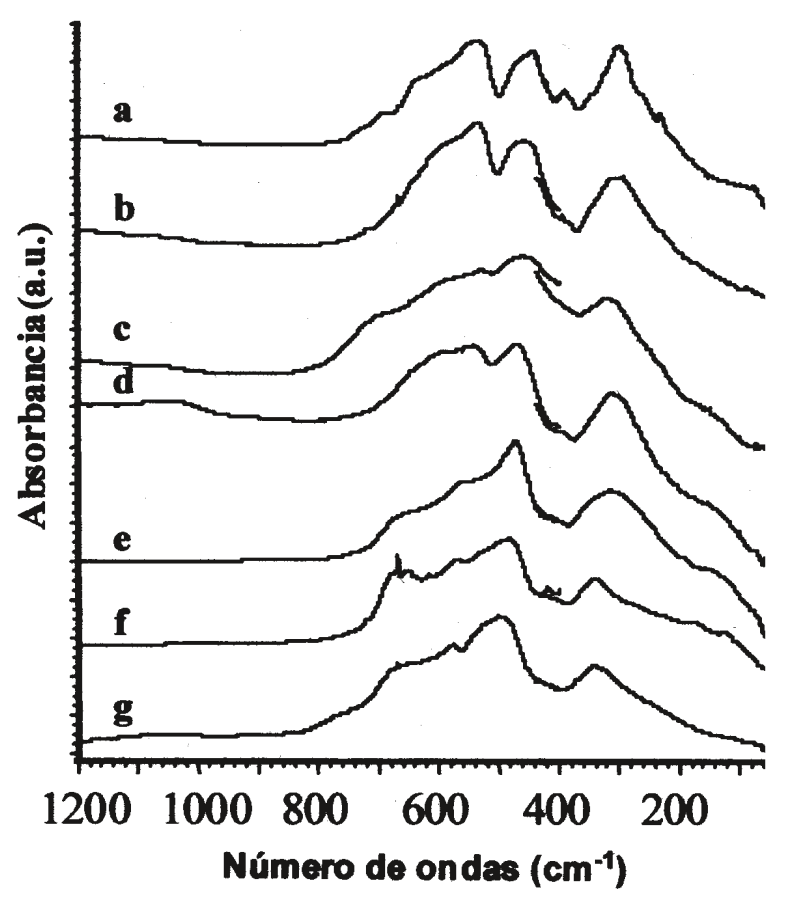

Fig. 2. Espectros FT-IR de las muestras calcinadas a $400{ }^{\circ} \mathrm{C}$. a) Fe2O3, b) Fe1.8Ga0.2Oy, c) Fe1.5Ga0.5Oy, d) Fe1.0Ga1.0Oy, e) Fe0.5Ga1.5Oy, f) $\mathrm{Fe} 0.2 \mathrm{Ga} 1.8 \mathrm{Oy}$ y g) $\mathrm{Ga}_{2} \mathrm{O}_{3}$.

Fe-Al (16) y se asoció con la progresiva sustitución de Fe por Ga en la estructura. Para la muestra con relación de composición $\mathrm{Fe} / \mathrm{Ga}=1$, el espectro es similar al de la muestra de Fe con tres características principales a 544, 470 y $322 \mathrm{~cm}^{-1}$. Mayores contenidos de Ga producen una marcada transformación aumentando la intensidad la banda a $470 \mathrm{~cm}^{-1}$ (Fig. 2,e) y comenzando a distinguirse las bandas atribuidas a la fase $\alpha-\mathrm{Ga}_{2} \mathrm{O}_{3}$. Todas estas características son apreciables en el espectro de la muestra $\mathrm{Fe}_{0.2} \mathrm{Ga}_{1.8} \mathrm{O}_{\text {y }}$ que es prácticamente análogo al espectro de la muestra de Ga. Este material presenta bandas de absorción próximas a 343, 497, 576, 668 y 775 (hombro) $\mathrm{cm}^{-1}$ en buen acuerdo con los espectros aportados por Burholder y col. (17).

Por tanto, los espectros FT-IR confirman la progresiva introducción del Ga en la estructura $\alpha-\mathrm{Fe}_{2} \mathrm{O}_{3}$ y la proximidad de los modos activos para ambos óxidos puros hace que en los óxidos mixtos se observen bandas cada vez más amplias, resultado del solapamiento de las bandas de los modos activos de los óxidos puros.

\subsubsection{ESPECTROSCOPÍA DE REFLECTANCIA DIFUSA VIS-UV.}

Como se puede observar en la Fig. 3, el espectro visible-ultravioleta de la muestra de Fe presenta una absorción principal a 352 $\mathrm{nm}$ con hombros adicionales próximos a 252, 450 y $515 \mathrm{~nm}$. Otras características más débiles se encuentran a más altas longitudes de ondas, próximas a 641 y $869 \mathrm{~nm}$. De acuerdo con previos estudios (18), las bandas de absorción a 252 y $515 \mathrm{~nm}$ pueden relacionarse razonablemente con las transferencias de carga $\mathrm{O}^{2-} \rightarrow \mathrm{Fe}^{3+}$ y $\mathrm{Fe}^{3+} \rightarrow \mathrm{Fe}^{3+}$ de $\mathrm{Fe}^{3+}$ en ambiente octaédrico, respectivamente. Mientras que las características a 641 y $869 \mathrm{~nm}$ son debidas a las transiciones $\mathrm{d} \rightarrow \mathrm{d},{ }^{4} \mathrm{~A}_{2}\left({ }^{4} \mathrm{~F}\right) \rightarrow{ }^{4} \mathrm{~T}_{2}\left({ }^{4} \mathrm{G}\right)$ y ${ }^{4} \mathrm{~A}_{2}\left({ }^{4} \mathrm{~F}\right) \rightarrow{ }^{4} \mathrm{~T}_{1}\left({ }^{4} \mathrm{G}\right)$, de $\mathrm{Fe}^{3+}$ también en ambiente octaédrico. Algunos autores asocian estas

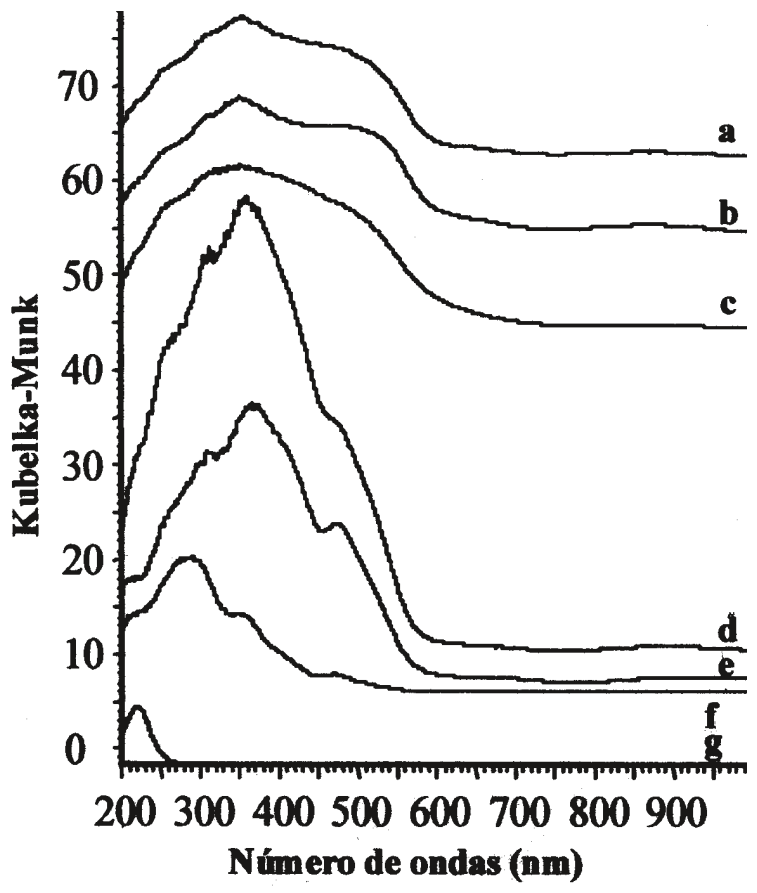

Fig. 3. Espectros electrónicos Vis-UV de las muestras calcinadas a $400{ }^{\circ} \mathrm{C}$. a) Fe2O3, b) Fe1.8Ga0.2Oy, c) Fe1.5Ga0.5Oy, d) Fe1.0Ga1.0Oy, e) $\mathrm{Fe} 0.5 \mathrm{Ga} 1.5 \mathrm{Oy}, \mathrm{f}) \mathrm{Fe} 0.2 \mathrm{Ga} 1.8 \mathrm{Oy}$ y g) $\mathrm{Ga}_{2} \mathrm{O}_{3}$.

bandas con $\mathrm{Fe}^{3+}$ situado en la superficie y por tanto la intensidad va a depender acusadamente del método de síntesis (12,19). Por otra parte, las bandas a 352 y $450 \mathrm{~nm}$ tienen una interpretación más compleja debido a que sus posiciones, forma e intensidad varían significativamente en función de la matriz de $\mathrm{Fe}^{3+}$. Teniendo en cuenta estas circunstancias, podrían corresponder a las transiciones $\mathrm{d} \rightarrow \mathrm{d},{ }^{4} \mathrm{~A}_{2}\left({ }^{4} \mathrm{~F}\right) \rightarrow{ }^{4} \mathrm{E}\left({ }^{4} \mathrm{D}\right) \mathrm{y}{ }^{6} \mathrm{~A}_{1} \rightarrow{ }^{4} \mathrm{~T}_{2}\left({ }^{4} \mathrm{D}\right)$, de Fe ${ }^{3+}$ en coordinación octaédrica y tetraédrica, respectivamente. La segunda asignación también se fundamenta en la tendencia de $\mathrm{Ga}^{3+}$ a ocupar posiciones tetraédricas, mucho más marcada en la estructura $\beta-\mathrm{Ga}_{2} \mathrm{O}_{3}(1)$, que puede influenciar la ocupación del $\mathrm{Fe}^{3+}$.

Este espectro se modifica sustancialmente con el aumento del contenido de $\mathrm{Ga}$, manifestando una amplia absorción próxima a $352 \mathrm{~nm}$ para la muestra de composición Fe/Ga = 3 (Fig. 3, c). Comparando con la muestra de Ga (Fig. 3,g), se puede deducir que las bandas de absorción del óxido de Ga no son importantes en el intervalo de estudio. De esta forma, los espectros se pueden interpretar mediante transiciones electrónicas que implican cationes $\mathrm{Fe}^{3+}$ en coordinación octaédrica más o menos influenciadas por el grado de ocupación de los cationes $\mathrm{Ga}^{3+}$. En realidad, algunos efectos de este tipo se han descrito para estructuras tipo espinela (20). Mayores aumentos del contenido de Ga incrementan notablemente la intensidad absoluta del espectro definiendo aún más las características descritas antes (Fig. 3,d). Sin embargo, la intensidad vuelve de nuevo a disminuir a partir de la muestra $\mathrm{Fe}_{0.5} \mathrm{Ga}_{1.5} \mathrm{O}_{\mathrm{y}}$ (Fig. 3,e) hasta no presentar prácticamente bandas de absorción en la muestra de Ga (Fig. 3,g). La banda situada a $237 \mathrm{~nm}$ se debe probablemente a trazas de algunos cationes no determinados de la sal precursora. Esta evolución de los espectros electrónicos con el contenido de Ga nos está indicando un efecto de dilución de los cationes $\mathrm{Fe}^{3+}$ por parte del Ga ${ }^{3+}$ que no presentan transiciones $\mathrm{d} \rightarrow \mathrm{d}$ y por tanto, la intensidad de las bandas disminuye notablemente. 


\subsection{Estudio de la superficie de las muestras}

\subsubsection{HIDROXILOS DE LA SUPERFICIE}

En la Fig. 4 se muestran los espectros de las muestras calcinadas a $400{ }^{\circ} \mathrm{C}$ en la zona de tensión de los hidroxilos después de tratar las muestras a $400{ }^{\circ} \mathrm{C}$, excepto la de Fe que se trató a 350 ${ }^{\circ} \mathrm{C}$. Después de realizar vacío a $350{ }^{\circ} \mathrm{C}$, se pueden distinguir hasta 6 bandas a 3684 (hombro), 3667, 3646, 3627, 3590 (hombro) y 3480 (hombro) $\mathrm{cm}^{-1}$ (Fig. 4,a). Las dos bandas a menores números de ondas desaparecen después de calentar a $400{ }^{\circ} \mathrm{C}$, mientras que las otras invierten la intensidad. Como aportaron previamente algunos autores $(15,21)$, estos grupos hidroxilo son típicos de la fase $\alpha-\mathrm{Fe}_{2} \mathrm{O}_{3}$, hematita, atribuidos a grupos $\mathrm{OH}$ estables (OH terminales sobre cationes octaédricos y punteados) y enlaces de hidrógeno posiblemente en "clusters", respectivamente. Pequeñas adiciones de Ga disminuyen significativamente la transmitancia en esta región, apareciendo el espectro muy perturbado (Fig. 4 b,c). Sin embargo todavía es posible distinguir tres grupos hidroxilo próximos a 3731, 3666 y $3647 \mathrm{~cm}^{-1}$, en parte, ya asignados. La banda a $3731 \mathrm{~cm}^{-1}$ se debe a la presencia de trazas de $\gamma-\mathrm{Fe}_{2} \mathrm{O}_{3}$, maghemita, concentrada sobre la superficie en buen acuerdo con la pobre cristalinidad de estos materiales encontrada en los difractogramas de rayos X. La fase $\gamma-\mathrm{Fe}_{2} \mathrm{O}_{3}$ es una impureza típica en esta clase de síntesis (15), aunque no fue identificada en DRX. Para la relación de composición Fe/ $\mathrm{Ga}=1$, el espectro vuelve a resolverse (Fig. 4, e) identificándose dos bandas a 3665 y 3646 (hombro) $\mathrm{cm}^{-1}$, además de otra más débil a $3540 \mathrm{~cm}^{-1}$ las cuales son todavía análogas a aquellas de la fase $\alpha-\mathrm{Fe}_{2} \mathrm{O}_{3}$ discutidas antes. Cuando el contenido de Ga es superior al de Fe (Fig. 4 e,f), la posición e intensidad de los grupos hidroxilos comienza a ser similar a los de la muestra de Ga. Esta superficie se caracteriza por dos tipos de $\mathrm{OH}$ bien definidos a 3690 y 3660 (más fuerte) $\mathrm{cm}^{-1}$ desplazados aproximadamente $20 \mathrm{~cm}^{-1}$ con respecto a los de la fase $\alpha-\mathrm{Fe}_{2} \mathrm{O}_{3}$. De acuerdo con estos resultados, se deduce que la introducción de Ga cambia significativamente la superficie de las muestras, estando en este constituida por grupos $\mathrm{OH}$ sobre cationes octaédricos y puenteados, los últimos en mayor porcentaje cualitativamente.

\subsubsection{ESTUDIO DE LA ACIDEZ DE LA SUPERFICIE}

La acidez de la superficie de las muestras se estudió mediante la adsorción de la molécula de pivalonitrilo sobre las pastillas prensadas de las muestras después de su activación a $400^{\circ} \mathrm{C}$. Los resultados obtenidos antes y después de realizar vacío a temperatura ambiente se muestran en las Fig. 5 y 6, respectivamente. La interacción del pivalonitrilo con la superficie se realiza mediante el par de electrones localizado en el nitrógeno del grupo $\mathrm{CN}$ identificándose una banda a $2235 \mathrm{~cm}^{-1}$ en la fase de vapor (Fig 5,a) (22). En contacto con la superficie de la muestra con Fe permanece prácticamente invariable en su posición y pierde intensidad, observándose este mismo comportamiento hasta la muestra $\mathrm{Fe}_{1.5} \mathrm{Ga}_{0.5} \mathrm{O}_{\mathrm{y}}$ (Fig. 5,b,c). A partir de este material aparece una banda adicional en torno a $2268 \mathrm{~cm}^{-1}$ que se desplaza hasta $2274 \mathrm{~cm}^{-1}$ con el aumento del contenido de Ga (Fig. $5, \mathrm{~d}-\mathrm{g}$ ). Las dos bandas son cada vez más intensas llegando a superar en intensidad la correspondiente a mayores números de onda y presentando dos máximos en la muestra pura de Ga (Fig. 5,h). Por comparación con el espectro del vapor de pivalonitrilo, estas bandas se asocian a pivalonitrilo fisisorbido $\left(2235 \mathrm{~cm}^{-1}\right)$ y a la interacción con un solo tipo centro ácido Lewis $\left(2274 \mathrm{~cm}^{-1}\right)$ excepto en el caso de la muestra de Ga que se encuentran dos (2278 y $\left.2268 \mathrm{~cm}^{-1}\right)$. Después de realizar vacío (Fig. 6), se observa

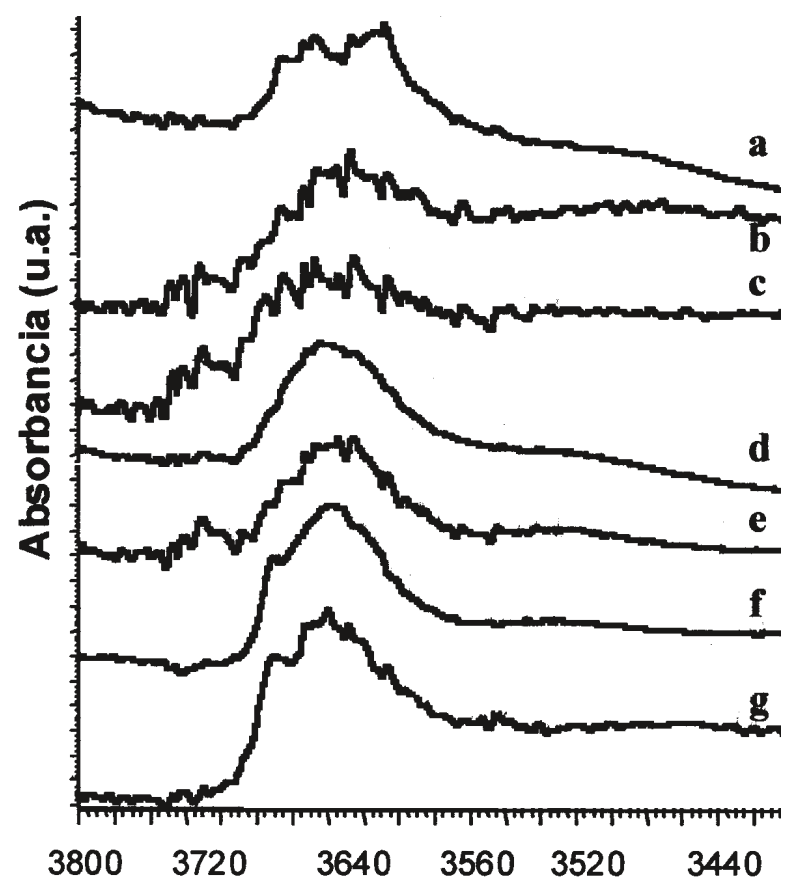

Número de ondas $\left(\mathrm{cm}^{-1}\right)$

Fig. 4. Espectros FT-IR en la región de tensión de los hidroxilos de las muestras después de activación a $350^{\circ} \mathrm{C}$ y evacuación. a) Fe2O3, b) Fe1.8Ga0.2Oy, c) Fe1.5Ga0.5Oy, d) Fe1.0Ga1.0Oy, e) Fe0.5Ga1.5Oy, f) $\mathrm{Fe} 0.2 \mathrm{Ga} 1.8 \mathrm{Oy}$ y g) $\mathrm{Ga}_{2} \mathrm{O}_{3}$.

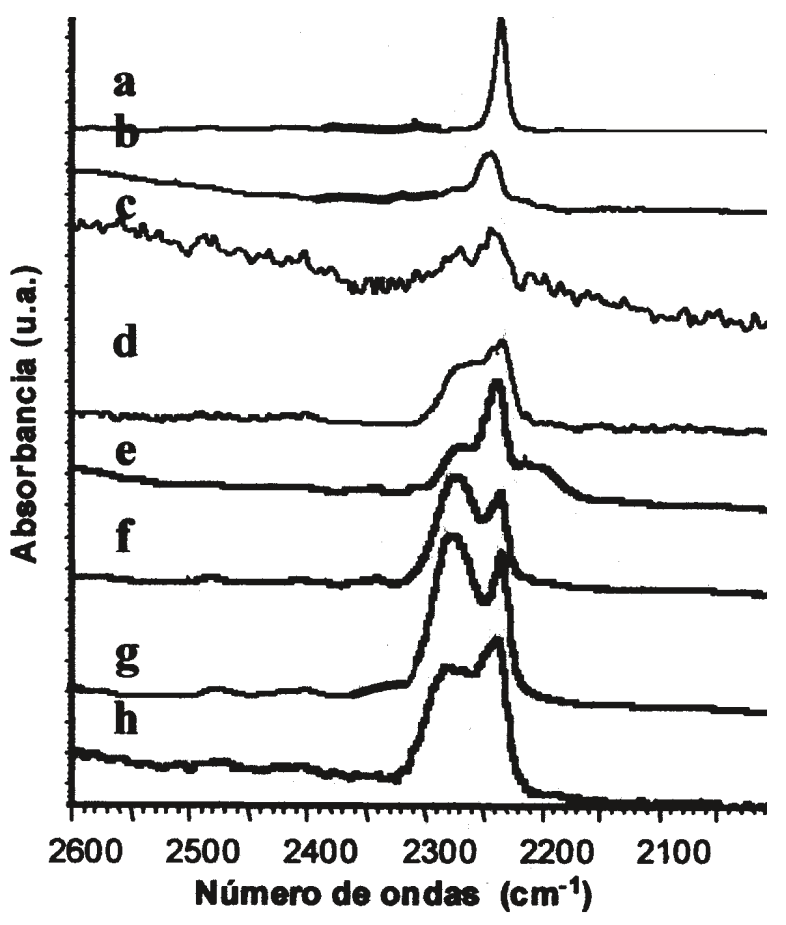

Fig. 5. Espectros FT-IR de las muestras en la zona $2000-2600 \mathrm{~cm}-1$ después de la activación de la pastilla y adsorción de pivalonitrilo a $20{ }^{\circ} \mathrm{C}$. a) Pivalonitrilo (vapor), b) Fe2O3, c) Fe1.8Ga0.2Oy, d) Fe1.5Ga0.5Oy, e) Fe1.0Ga1.0Oy, f) Fe0.5Ga1.5Oy, g) Fe0.2Ga1.8Oy y h) $\mathrm{Ga}_{2} \mathrm{O}_{3}$. 


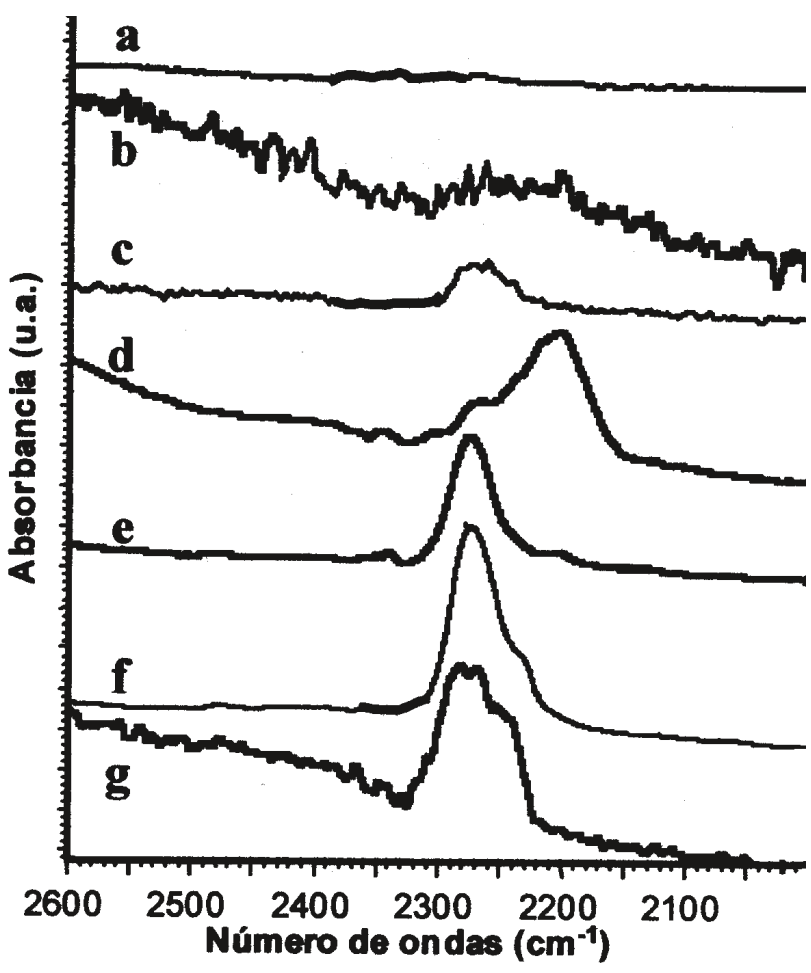

Fig. 6. Espectros FT-IR de las muestras en la zona $2000-2600 \mathrm{~cm}^{-1}$ después de la activación de la pastilla y adsorción de pivalonitrilo y evacuación a $20^{\circ} \mathrm{C}$. a) Fe2O3, b) Fe1.8Ga0.2Oy, c) Fe1.5Ga0.5Oy, d) Fe1.0Ga1.0Oy, e) Fe0.5Ga1.5Oy, f) Fe0.2Ga1.8Oy y g) $\mathrm{Ga}_{2} \mathrm{O}_{3}$.

que prácticamente no quedan moléculas sobre la superficie para las muestras con bajo contenido de Ga (Fig. 6,a,b,c), mientras que las muestras de alto contenido de Ga conservan la banda 2274 $\mathrm{cm}^{-1}$ más intensa cada vez y con un pequeño hombro a $2238 \mathrm{~cm}^{-1}$ para las composiciones $\mathrm{Fe}_{0.5} \mathrm{Ga}_{1.5} \mathrm{O}_{\text {y }}$ y $\mathrm{Fe}_{0.2} \mathrm{Ga}_{1.8} \mathrm{O}_{\mathrm{y}}$. Además se encuentra un comportamiento especial para la muestra $\mathrm{Fe}_{1.0} \mathrm{Ga}_{1.0} \mathrm{O}_{\mathrm{y}}$ donde aparece una banda adicional a $2201 \mathrm{~cm}^{-1}$. Posteriores aumentos de temperatura producen la desorción progresiva del pivalonitrilo hasta desaparecer las bandas anteriores a $150{ }^{\circ} \mathrm{C}$.

Basándose en estos datos se puede concluir que las muestras presentan mayor acidez de Lewis con el aumento del contenido de Ga situándose la banda a $2274 \mathrm{~cm}^{-1}$ entre la acidez observada para el $\mathrm{V}_{2} \mathrm{O}_{5}\left(2280 \mathrm{~cm}^{-1}\right)$ y el $\mathrm{TiO}_{2}$ anatasa $\left(2265 \mathrm{~cm}^{-1}\right)$, pero los centros ácidos son débiles porque en el intervalo de temperaturas $150-200^{\circ} \mathrm{C}$ todas las móleculas desorben de la superficie (23). Por otra parte el tipo de adsorción es vertical ya que no se observa ninguna perturbación en la zona de tensión y deformación de los $\mathrm{CH}$. Finalmente, comentar que el desplazamiento observado en la banda de tensión de los $\mathrm{OH}$ desde $3600 \mathrm{~cm}^{-1}$ hasta casi $3300 \mathrm{~cm}^{-1}$ con el aumento del contenido de Ga, está indicando que la acidez existente en estas superficies es también de tipo Brönsted y es mayor con el contenido de Ga. Este desplazamiento depende de la fuerza del puente de hidrógeno formado entre el $\mathrm{OH}$ de la superficie y el grupo $\mathrm{CN}$ (23).

\subsubsection{ADSORCIÓN DE METANOL SOBRE LA SUPERFICIE}

En las Fig. 7 y 8 se muestran como ejemplo los espectros FT-IR obtenidos después de la adsorción de metanol sobre la muestra $\mathrm{Fe}_{1.8} \mathrm{Ga}_{0.2} \mathrm{O}_{\mathrm{y}}$ a temperatura ambiente y en evacuación a distintas temperaturas. A temperatura ambiente, se observa

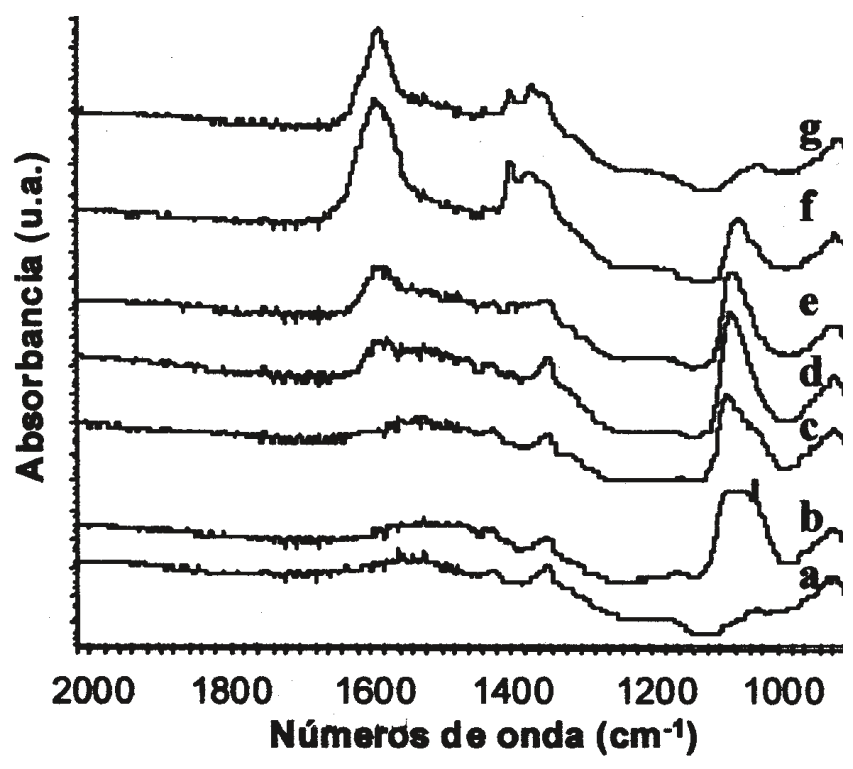

Fig. 7. Espectros FT-IR de la muestra $\mathrm{Fe}_{1.8} \mathrm{Ga}_{0.2} \mathrm{Oy}$ en la zona 900-2000 $\mathrm{cm}^{-1}$ después de la adsorción de metanol y evacuación a distintas temperaturas. a) Pastilla activada, b) $20{ }^{\circ} \mathrm{C}$, c) ev. $+100{ }^{\circ} \mathrm{C}$, d) ev. $+150{ }^{\circ} \mathrm{C}$, e) ev. $\left.+200{ }^{\circ} \mathrm{C}, \mathrm{f}\right)$ ev. $+250{ }^{\circ} \mathrm{C}$ y g) ev. $+300^{\circ} \mathrm{C}$.

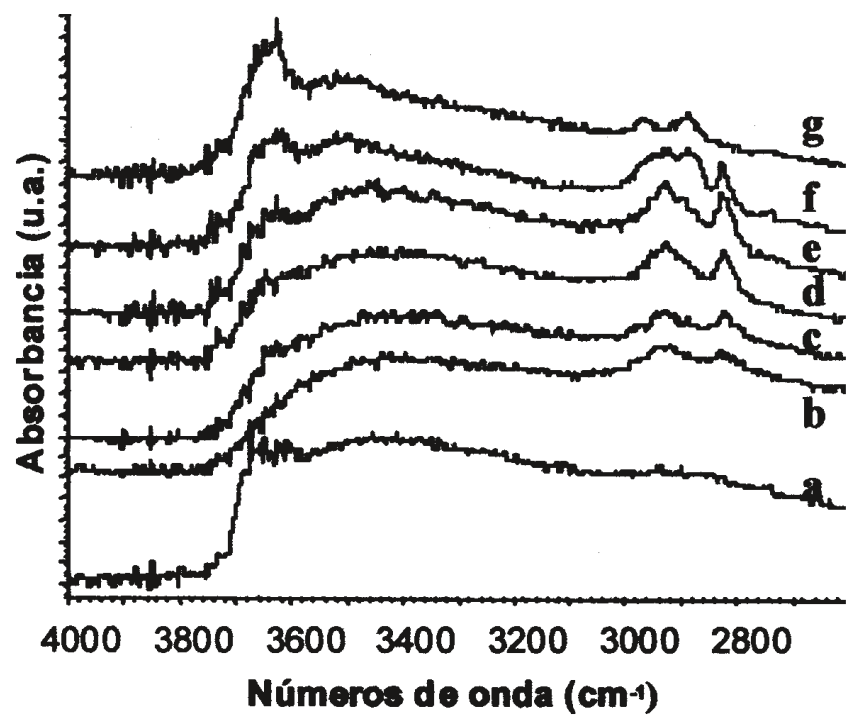

Fig. 8. Espectros FT-IR de la muestra $\mathrm{Fe}_{1.8} \mathrm{Ga}_{0.2} \mathrm{Oy}$ en la zona 2600-4000 $\mathrm{cm}^{-1}$ después de la adsorción de metanol y evacuación a distintas temperaturas. a) Pastilla activada, b) $20{ }^{\circ} \mathrm{C}, \mathrm{c}$ ) ev. $+100{ }^{\circ} \mathrm{C}, \mathrm{d}$ ) ev. $+150{ }^{\circ} \mathrm{C}$, e) ev. $\left.+200{ }^{\circ} \mathrm{C}, \mathrm{f}\right)$ ev. $+250{ }^{\circ} \mathrm{C}$ y g) ev. $+300^{\circ} \mathrm{C}$.

un doblete a 1033 y $1069 \mathrm{~cm}^{-1}$ (24) y una banda a $1365 \mathrm{~cm}^{-1}$ (Fig. $7, a)$ que corresponden a la $\delta \mathrm{OH}$ del metanol en fase vapor e interaccionando con la superficie y a $\mathrm{vOH}$ de la misma molécula. Mientras que en la zona de $2600-4000 \mathrm{~cm}^{-1}$ los hidroxilos de la superficie apenas se han modificado. En esta zona también se sitúan las tensiones $v \mathrm{CH}$ próximas a 2800 y $2900 \mathrm{~cm}^{-1}$. Un ligero aumento de la temperatura provoca la desaparición de la banda a $1034 \mathrm{~cm}^{-1}$, mientras que la de $1067 \mathrm{~cm}^{-1}$ va desapareciendo progresivamente, a la vez que aparece un doblete (1360 y $1370 \mathrm{~cm}^{-1}$ ) y una banda muy aguda a $1590 \mathrm{~cm}^{-1}$ debidas a la formación de especies formiato $\left(v_{\mathrm{as}} \mathrm{COO}, \delta \mathrm{CH}\right.$ y $\left.v_{\mathrm{s}} \mathrm{COO}\right)$ (25). En paralelo la zona de los hidroxilos de la superficie forma una banda amplia que se desplaza hasta $3400 \mathrm{~cm}^{-1}$, indicando la formación incial de puentes de hidrógeno y la poste- 
rior desprotonación dando especies metóxido. Después de un tratamiento a $350{ }^{\circ} \mathrm{C}$ todo el metanol se ha transformado en carbonatos y $\mathrm{CO}_{2}$ (Fig. 7,g) y la zona de los hidroxilos se ha regenerado (Fig. 8,g). El comportamiento observado con el resto de las composiciones muestra que la interacción es más fuerte con el aumento del contenido de Ga apareciendo la banda de $\delta \mathrm{OH}$ del metanol a mayores números de ondas. No obstante la reactividad aumenta para bajos contenidos de Ga encontrándose la mayor actividad para la muestra $\mathrm{Fe}_{1.8} \mathrm{Ga}_{0.2} \mathrm{O}_{\mathrm{y}}$ donde los formiatos aparacen ya a $150{ }^{\circ} \mathrm{C}$.

\section{CONCLUSIONES}

Las conclusiones más importantes encontradas en este trabajo se pueden resumir en las siguientes:

- Los óxidos mixtos de Fe y Ga preparados por coprecipitación a $\mathrm{pH}$ controlado forman una disolución sólida en todo el intervalo de composiciones Fe/ Ga.

- La estabilidad de estas disoluciones se extiende hasta la temperatura de $800{ }^{\circ} \mathrm{C}$ y una relación de composiciones $\mathrm{Fe} / \mathrm{Ga}=$ 3 , debido, fundamentalmente, a la transición de fase $\alpha-\beta$ del óxido de Ga que da lugar a una estructura diferente.

- Los espectros electrónicos muestran que la adición de $\mathrm{Ga}^{3+}$ provoca la dilución de los cationes $\mathrm{Fe}^{3+}$ y una disminución de la intensidad de las bandas de las transiciones electrónicas.

- Los espectros FT-IR manifiestan la existencia de las disoluciones sólidas ensanchando las bandas como consecuencia del solapamiento de las bandas correspondientes a los óxidos Ga e Fe.

- La superficie de estos materiales está formada por grupos $\mathrm{OH}$ sobre cationes octaédricos y puenteados, fundamentalmente, siendo cualitativamente mayoritarios los últimos y desplazándose hacia mayores números de ondas con el aumento del contenido de Ga.

- La acidez de los óxidos mixtos es tipo Lewis débil aumentando la fortaleza de los centros a mayores contenidos de Ga y situándose entre la encontrada para $\mathrm{V}_{2} \mathrm{O}_{5}\left(2280 \mathrm{~cm}^{-1}\right)$ y el $\mathrm{TiO}_{2}$ anatasa $\left(2265 \mathrm{~cm}^{-1}\right)$. También se observa acidez de Brönsted manifestada en él desplazamiento de la banda en torno a $3600 \mathrm{~cm}^{-1}$ de los grupos hidroxilos hasta $3300 \mathrm{~cm}^{-1}$ en el $\alpha-\mathrm{Ga}_{2} \mathrm{O}_{3}$ como consecuencia de la formación de puentes de hidrógeno.

- La adsorción de metanol manifiesta la mayor reactividad de las muestras con bajo contenido de Ga, observándose la conversión de metanol a formiato a $150{ }^{\circ} \mathrm{C}$. Esto supone casi 80 ${ }^{\circ} \mathrm{C}$ de diferencia respecto al resto de las muestras.

\section{AGRADECIMIENTOS}

Los autores agradecen la financiación de este trabajo a la OTAN (CRG-960316) y a la Junta de Castilla y León (SA37/98). JMGA agradece, en especial, al MEC la concesión de una beca FPI, postdoctoral.

\section{BIBLIOGRAFÍA}

1. V. Kanazirev, R. Dimitrova, G.L. Price,A. Yu Khodakov, L.M. Kustov, V.B Kazansky. "Characterization of Some Bifunctional Catalysts for oil Refining and Petrochemistry by means of Model Reactions" J. Mol. Catal. 70 111-117 (1990).

2. V. Kanazirev, G.L. Price,K.M. Dooley. "Enhancement in Propane Aromatization with $\mathrm{Ga}_{2} \mathrm{O}_{3} /$ HZSM-5 Catalysts" J. Chem. Soc., Chem. Commun. 712-713 (1990).

3. P. Mèriaudeau, S.B.A. Hamid, C. Naccache. "Propane Conversion on Ga-HZSM-5: Effect of Aging on the Dehydrogenating and Acid Functions using Pyridine as an IR Probe" J. Catal. 139 679-682 (1993).

4. E. Lalik, X. Liu, J. Klinowski. "The Role of gallium in the Cat alytic Activity of Zeolite [Si,Ga]-ZSM-5 for Metanol Conversion" J. Phys. Chem. 96 805-809 (1992).

5. Yu Kodakov, L.M. Kustov, T.N. Bondarenko, A.A. Dergachev, V.B. Kazansky, Kh.M. Minachev, G. Borbely, H.K. Beyer. "Investigation of the Different States of Galliumin Crystilline Galosilicates witn Pentasil Strucutre and their Role in Propane Aromatization" Zeolites 10 603-7 (1990)

6. G.L. Price, V. Kanazirev. "Gallium Oxide/HZSM-5 Propane Aromatization Catalysts: Formation of Active Centers via Solid-state Reaction" J. Catal. 126 267-278 (1990)

7.K. Shimizu, A. Satsuma, T. Hattori. "Selective Catalytic reduction of NO by Hydrocarbons on $\mathrm{Ga}_{2} \mathrm{O}_{3}-\mathrm{Al}_{2} \mathrm{O}_{3}$ Catalysts" App. Catal. B: Environ. 16 319-326 (1998).

8. M. Baldi, V. Sanchez Escribano, J.M. Gallardo-Amores, F. Milella, G. Busca. "Characterization of Manganese and Iron Oxides as Combustion Catalysts for Propane and Propene" Appl. Catal. B: Environ. 17, L-175- L182 (1998).

9. V. Sánchez Escribano, J.M. Gallardo Amores, G. Busca, R. Ruano Casero, E. Pérez Bernal. "Estudio Morfológico-estructural del Sistema Mixto de $\mathrm{Mn}_{2} \mathrm{O}_{3}$ $\mathrm{Al}_{2} \mathrm{O}_{3}$ " "XXIII Reunión Ibérica de Adsorçao", Evora, (Portugal), Abstracts book, p. 35-38 (1998)

10. J.M. Gallardo-Amores, V. Sánchez-Escribano, G. Busca. “Characterization of Fe Ga Mixed Hydroxides Powders" J. Mater. Chem. 9(5) 1161-1166 (1999).

11. B.G. Hyde, S. Andersson. "Inorganic Crystal Structures", 1st edn., Wiley Intersience, New York (1989)

12. G. Busca, G. Ramis, M.C. Prieto, V. Sànchez Escribano. “Preparation and Characterization of $\mathrm{Fe}_{2-x} \mathrm{Cr}_{x} \mathrm{O}_{3}$ Mixed Oxide" J. Mater. Chem. 3 665-673 (1993).

13. Baraton, G. Busca, M.C. Prieto, G. Ricchiardi, V. Sánchez Escribano. “On the Vibrational Spectra and Structure of $\mathrm{FeCrO}_{3}$ and of the Ilmenite-type Compounds $\mathrm{CoTiO}_{3}$ and $\mathrm{NiTiO}_{3}{ }^{\prime \prime}$ J. Solid State Chem. 112 9-14 (1994).

14. C.J. Serna, J.L. Rendon, J. E. Iglesias. "Infrared Surface Modes in Corundumtype Microcrystalline Oxides" Spectrochim. Acta. 38A 797-802 (1982)

15. M.C. Prieto, J.M. Gallardo-Amores, V. Sanchez Escribano, G. Busca. "Characterization of Coprecipitated $\mathrm{Fe}_{2} \mathrm{O}_{3}-\mathrm{Al}_{2} \mathrm{O}_{3}$ Powders" J. Mater. Chem. 4 1123-1130 (1994)

16. V. Sánchez Escribano, J. M. Gallardo Amores, E. Finocchio, M. Daturi, G. Busca. "Characterization of $\alpha-(\mathrm{Fe}, \mathrm{Al})_{2} \mathrm{O}_{3}$ Solid-solution Powders" J. Mater. Chem. 5 1943-1951 (1995).

17. T.R. Burkholder, T.J. Yustein y L.J. Andrews. "Reactions of Pulsed Laser Evaporated Ga and In Atoms with Molecular Oxygen. Matrix Infrared Spectra of New $\mathrm{GaO}_{2}$ and $\mathrm{InO}_{2}$ Species" J. Phys. Chem. 96 10189-10195 (1992).

18. P.G. Manning. "Charge-transfer interactions and the origin of color in brown vesuvianite" Canadian Mineral. 15 508-511 (1977.

19. G. Busca, V. Lorenzelli, G. Ramis, R. Willey, "Surface Sites on High-area Spinel-type Metal Oxides" Langmuir 9 1492-1499 (1993).

20. M. Lenglet, M. Bizi, C.K. Jorgensen. “Influence de la Distribution Cationique et de la Nature des Interactions Magnetiques sur l'intensite des Transitions de Paires de l'ion $\mathrm{Fe}^{3+}$ dans quelques Spinelles Mixtes" J. Solid State Chem. 86 82-87 (1990).

21. V. Lorenzelli, G. Busca. "Infrared studies of the surface of $\alpha-\mathrm{Fe}_{2} \mathrm{O}_{3}$ " Mater. Chem. Phys. 13 261-281 (1985)

22. C.U. I. Odenbrand, J.G.M. Brandin, G. Busca. “Surface acidity of silica-titania mixed oxides" J. Catal. 135 505-517 (1992).

23. G. Busca. "Spectroscopic Characterisation of the Acid Properties of Metal Oxide Catalysts" Catal. Today 41 191-206 (1998).

24. J.P. Gallas, Tesis doctoral, Universidad de Caen (1984).

25. G. Busca, P. Forzatti, J.C. Lavalley, E. Tronconi. “A TPD, FT-IR and Catalytic Study of the Interaction of Methanol with Pure and $\mathrm{KOH}$ doped $\mathrm{TiO}_{2}$ Anatase" in "Catalysis by Acids and Bases", B. Imelik et al. Eds., Elsevier Science, p. 15-24, The Nethenlands (1985). 